\title{
Survival benefits from splenic hilar lymph node dissection by splenectomy in gastric cancer patients: relative comparison of the benefits in subgroups of patients
}

\author{
Toshiyuki Kosuga $\cdot$ Daisuke Ichikawa $\cdot$ Kazuma Okamoto $\cdot$ \\ Shuhei Komatsu $\cdot$ Atsushi Shiozaki $\cdot$ Hitoshi Fujiwara • \\ Eigo Otsuji
}

Received: 23 September 2010/Accepted: 27 December 2010/Published online: 18 February 2011

(c) The International Gastric Cancer Association and The Japanese Gastric Cancer Association 2011

\begin{abstract}
Background The present study estimated survival benefits from lymph node dissection at the splenic hilus in advanced proximal gastric cancer patients who underwent total gastrectomy with simultaneous splenectomy, and then determined patient subgroups that received relatively high survival benefits from splenectomy.

Methods A total of 280 patients with advanced proximal gastric cancer who underwent curative total gastrectomy with simultaneous splenectomy were retrospectively analyzed. Patients with primary tumors directly invading the spleen or pancreas and those with gross metastases to the para-aortic nodes, as determined by intraoperative diagnosis, were excluded from analyses. The index of estimated benefit from lymph node dissection at the splenic hilus by splenectomy was calculated for each clinicopathological factor by multiplying the incidence of splenic hilar metastasis by the 5-year survival rate of patients with metastasis to that nodal station.

Results Thirty patients (10.7\%) showed lymph node metastasis at the splenic hilus, and the 5-year survival rate of these patients was $51.3 \%$ (overall index 5.49). The index was relatively high in patient subgroups with tumors localized on the greater curvature (19.4) and Borrmann type 4 cancers (12.9), while relatively low in subgroups with encircling tumors (1.62) and tumors invading adjacent organs other than the spleen and pancreas (0).
\end{abstract}

T. Kosuga and D. Ichikawa contributed equally to this work.

T. Kosuga · D. Ichikawa $(\bowtie) \cdot$ K. Okamoto $\cdot$ S. Komatsu •

A. Shiozaki · H. Fujiwara · E. Otsuji

Division of Digestive Surgery, Department of Surgery,

Kyoto Prefectural University of Medicine, 465 Kajii-cho,

Kamigyo-ku, Kyoto 602-8566, Japan

e-mail: ichikawa@koto.kpu-m.ac.jp
Conclusion Patients with tumors localized on the greater curvature and Borrmann type 4 cancers might obtain relatively high survival benefits from lymph node dissection at the splenic hilus by splenectomy.

Keywords Gastric cancer · Surgery · Splenectomy · Lymph nodes · Prognosis

\section{Introduction}

Gastrectomy with lymph node dissection is the standard treatment for gastric cancer, but the optimal extent of lymph node dissection remains controversial [1-5]. In gastric cancer involving the proximal third of the stomach, lymph node metastases to the splenic hilus occur as cancer progresses [6-13]. Therefore, the Japanese Gastric Cancer Association (JGCA) recommends total gastrectomy with simultaneous splenectomy to facilitate dissection of these possible lymph node metastases in advanced proximal gastric cancer [14]. In fact, some gastric cancer patients with pathologically proven metastasis at the splenic hilus have been cured completely by simultaneous splenectomy at total gastrectomy. Many recent reports, however, demonstrated no survival benefit from splenectomy, and conversely higher postoperative morbidity and mortality [10-12, 15-17]. In addition, the potential role of the spleen in the host immune system has recently been emphasized [18]. Therefore, it remains controversial whether routine splenectomy during total gastrectomy should be performed for patients with advanced proximal gastric cancer.

The survival benefits from splenectomy, however, would differ among patient subgroups with each clinicopathological factor that is correlated with the incidence of lymph node metastasis at the splenic hilus and prognosis. 
Sasako et al. [5] proposed a simple and practical method to evaluate the therapeutic value of dissecting each lymph node station, in which the index for the efficacy of dissecting a lymph node station is estimated by multiplying the incidence of metastasis to a particular lymph node station by the 5-year survival rate of patients with metastasis to that station. In the present study, the survival benefits of splenectomy for patients with advanced proximal gastric cancer were estimated by calculating this index for the splenic hilar nodes, and subgroups that may benefit from splenectomy were identified.

\section{Materials and methods}

\section{Patients}

Between 1974 and 2006, a total of 2,599 patients with histologically proven primary gastric cancer underwent surgical resection at the Kyoto Prefectural University of Medicine, Japan. Of these, 280 patients with advanced gastric cancer involving the proximal third of the stomach who underwent curative total gastrectomy with simultaneous splenectomy (pancreaticosplenectomy in 91 patients and pancreas-preserving splenectomy in 189 patients) were enrolled in this retrospective study. In this series, there was no difference in the distribution of cases during each decade. The patient population was composed of 181 males and 99 females with a mean patient age of 58.7 years (range 24-80). Patients with primary tumors directly invading the spleen or pancreas and those with gross metastases to the para-aortic nodes, as determined by intraoperative diagnosis, were excluded from analyses. Patients who had shown macroscopically suspicious lymph nodes at the splenic hilus were included in the analyses because we do not have any data on the macroscopic diagnosis of metastasis to that nodal station in cases before 1995. None of the patients in the present study received preoperative chemotherapy and/or radiation therapy. All postoperative deaths, including death from surgical complications and causes other than cancer, were included in the survival analysis.

\section{Clinicopathological features}

Various clinicopathological features of the patients, including age, gender, Borrmann type, location of primary tumor, tumor size, histological type, depth of invasion, presence of metastatic lymph nodes, stage and curability of resection were retrospectively analyzed using the hospital database. Histopathological examinations were performed on primary lesions using serial sections to determine the depth of cancer invasion and other histological features by experienced pathologists. One section per node was examined for every dissected node to confirm the presence of metastasis. The macroscopic and microscopic classification of primary tumors and curability of resection were based on the JCGC staging system [14]. Depth of tumor invasion was classified as follows: MP (tumor invasion of muscularis propria), SS (tumor invasion of subserosa), SE (tumor penetration of serosa) and SI (tumor invasion of adjacent structures). The present study included 28 patients with primary tumors invading adjacent organs: 15 patients with invasion of the transverse colon, 2 patients with invasion of the liver and 11 patients with invasions of other organs, such as the diaphragm, adrenal gland and/or gallbladder. Primary tumor deeper than the muscularis propria was classified as advanced gastric cancer. Histological type of primary tumors was categorized into two groups: differentiated (well differentiated, moderately differentiated or papillary) or undifferentiated (poorly differentiated, signet-ring cell or mucinous). We classified the primary tumors into type 4 and non-type 4 based on the macroscopic appearance, because some previous studies have reported that splenic hilar metastasis was correlated with the Borrmann type of the primary tumor [7, 9].

\section{Surgical technique and postoperative follow-up}

At our institute, as in most Japanese hospitals, total gastrectomy, Japanese-style D2 lymph node dissection with pancreas-preserving splenectomy, and Roux-en-Y reconstruction with an esophagojejunostomy has mainly been performed for the treatment of advanced gastric cancers involving the proximal third of the stomach because the current JGCA guidelines recommend standardized D2 lymphadenectomy [14]. Prophylactic pancreaticosplenectomy was mainly performed before 1995, and pancreaspreserving splenectomy replaced pancreaticosplenectomy for complete D2 lymphadenectomy after 1995 unless carcinomas directly invade the pancreas. Prophylactic paraaortic nodal dissections were performed for 28 patients. Postoperative adjuvant chemotherapy was performed for 66 patients. The regimens were based on 5-fluorouracil, such as S-1, 5FU+MTX, or 5FU+CDDP. The follow-up program consisted of physical examination, hematological and blood chemistry panels, and blood tests for CEA and CA19-9. These assessments were repeated every 3 months for the first postoperative year and every 6 months thereafter for at least 5 years. Abdominal computed tomography (CT) and chest radiography were performed every 6 months. Endoscopy was performed annually to screen for cancers at the anastomotic site or remnant stomach, beginning 1 year after surgery. Positron emission tomography (PET) was performed at the patient's request and/or 
as an additional examination if occult elevation of serum tumor markers was detected.

Index of estimated benefits from lymph node dissection at the splenic hilus by splenectomy

The index of estimated benefit from lymph node dissection at the splenic hilus by splenectomy was calculated for each clinicopathological factor by multiplying the incidence of splenic hilar metastasis by the 5-year survival rate of patients with metastasis to that nodal station [5].

Statistical analysis

All statistical analyses were conducted using StatView version 5.0 (SAS Institute, Cary, NC, USA). The chisquare test was used to evaluate the differences between proportions for clinicopathological variables. Survival curves were constructed by the Kaplan-Meier method and differences in survivals were examined by log-rank test. All $p$ values $<0.05$ were considered significant.

\section{Results}

The incidence of lymph node metastasis at the splenic hilus

Of 280 patients with advanced proximal gastric cancer who underwent curative total gastrectomy with simultaneous splenectomy, 30 patients $(10.7 \%)$ had lymph node metastasis at the splenic hilus.

\section{Survival and mortality rate}

The 5-year survival rate of 280 patients who underwent curative total gastrectomy with simultaneous splenectomy was $43.0 \%$. There was no significant difference in survival between patients with splenic hilar metastasis and those without such metastasis (51.3 and $42.1 \%$, respectively). Postoperative adjuvant chemotherapy did not have a significant influence on the survival of patients analyzed in the present study (data not shown). Seven patients died from surgical complications, and the mortality rate was $2.50 \%$ (pancreas-preserving splenectomy; 3/189, 1.59\%, and pancreaticosplenectomy; 4/91, 4.40\%, respectively).

Correlations between clinicopathological variables and splenic hilar metastasis

Correlations between clinicopathological variables and splenic hilar metastasis are shown in Table 1. Borrmann type 4 cancers were more likely to show splenic hilar
Table 1 Correlations between clinicopathological variables and splenic hilar metastasis in patients undergoing curative gastrectomy with splenectomy

\begin{tabular}{|c|c|c|c|}
\hline \multirow[t]{2}{*}{ Variables } & \multicolumn{2}{|c|}{$\begin{array}{l}\text { Lymph node metastasis } \\
\text { at the splenic hilus }\end{array}$} & \multirow[t]{2}{*}{$p$ value } \\
\hline & $\begin{array}{l}\text { Positive } \\
(n=30)\end{array}$ & $\begin{array}{l}\text { Negative } \\
(n=250)\end{array}$ & \\
\hline Sex & & & 0.8738 \\
\hline Male & 19 & 162 & \\
\hline Female & 11 & 88 & \\
\hline Age (years) & & & 0.1147 \\
\hline$<65$ & 14 & 154 & \\
\hline$\geq 65$ & 16 & 96 & \\
\hline Borrmann type & & & $<0.0001 *$ \\
\hline Non-type 4 & 11 & 197 & \\
\hline Type 4 & 19 & 53 & \\
\hline Circumferential localization & & & $0.0317 *$ \\
\hline Non-greater curvature & 13 & 168 & \\
\hline Greater curvature & 6 & 25 & \\
\hline Encircling & 11 & 57 & \\
\hline Tumor size $(\mathrm{cm})$ & & & 0.4939 \\
\hline$<10$ & 22 & 196 & \\
\hline$\geq 10$ & 8 & 54 & \\
\hline Histological type & & & 0.4802 \\
\hline Differentiated & 12 & 117 & \\
\hline Undifferentiated & 18 & 133 & \\
\hline Depth of invasion & & & 0.4178 \\
\hline MP & 1 & 30 & \\
\hline SS & 9 & 71 & \\
\hline SE & 18 & 123 & \\
\hline SI & 2 & 26 & \\
\hline
\end{tabular}

metastasis than non-type 4 cancers $(p<0.0001)$. In addition, primary tumors involving the greater curvature (localized on the greater curvature and encircling) were more likely to metastasize to the splenic hilar nodes than tumors located in areas other than the greater curvature $(p=0.0098)$.

Index of estimated benefits from lymph node dissection at the splenic hilus

The overall index of estimated benefits from lymph node dissection at the splenic hilus in patients who underwent curative total gastrectomy with simultaneous splenectomy was 5.49 (incidence 0.107 , 5-year survival 51.3\%). The index of estimated benefits from lymph node dissection at the splenic hilus with respect to each clinicopathological factor is shown in Table 2. MP tumors were excluded from survival analysis because there was only one MP patient 
Table 2 Index of estimated benefits from dissection of splenic hilar lymph nodes in subgroups with each

clinicopathological factor a The index was estimated by multiplying the incidence of lymph node metastasis at the splenic hilus by the 5-year survival rate of patients with metastasis to that nodal station

b MP tumors were excluded from survival analysis because there was only one MP patient with splenic hilar metastasis

\begin{tabular}{|c|c|c|c|}
\hline Clinicopathological factor & $\begin{array}{l}\text { Incidence of lymph } \\
\text { node metastasis }\end{array}$ & $\begin{array}{l}\text { 5-year } \\
\text { survival }(\%)\end{array}$ & Index $^{a}$ \\
\hline \multicolumn{4}{|l|}{ Sex } \\
\hline Male & $0.105(19 / 181)$ & 55.3 & 5.81 \\
\hline Female & $0.111(11 / 99)$ & 44.4 & 4.93 \\
\hline \multicolumn{4}{|l|}{ Age } \\
\hline$<65$ & $0.0833(14 / 168)$ & 58.3 & 4.86 \\
\hline$\geq 65$ & $0.143(16 / 112)$ & 45.8 & 6.55 \\
\hline \multicolumn{4}{|l|}{ Borrmann type } \\
\hline Non-type 4 & $0.0529(11 / 208)$ & 56.3 & 2.98 \\
\hline Type 4 & $0.264(19 / 72)$ & 48.9 & 12.9 \\
\hline \multicolumn{4}{|l|}{ Circumferential localization } \\
\hline Non-greater curvature & $0.0718(13 / 181)$ & 64.6 & 4.64 \\
\hline Greater curvature & $0.194(6 / 31)$ & 100 & 19.4 \\
\hline Encircling & $0.162(11 / 68)$ & 10.0 & 1.62 \\
\hline \multicolumn{4}{|l|}{ Tumor size $(\mathrm{cm})$} \\
\hline$<10$ & $0.101(22 / 218)$ & 63.6 & 6.42 \\
\hline$\geq 10$ & $0.129(8 / 62)$ & 16.7 & 2.15 \\
\hline \multicolumn{4}{|l|}{ Histological type } \\
\hline Differentiated & $0.0930(12 / 129)$ & 55.6 & 5.17 \\
\hline Undifferentiated & $0.119(18 / 151)$ & 48.6 & 5.78 \\
\hline \multicolumn{4}{|l|}{ Depth of invasion } \\
\hline MP & $0.0323(1 / 31)$ & $-{ }^{\mathrm{b}}$ & $-{ }^{\mathrm{b}}$ \\
\hline SS & $0.113(9 / 80)$ & 62.5 & 7.06 \\
\hline $\mathrm{SE}$ & $0.128(18 / 141)$ & 44.9 & 5.75 \\
\hline SI & $0.0714(2 / 28)$ & 0 & 0 \\
\hline Overall & $0.107(30 / 280)$ & 51.3 & 5.49 \\
\hline
\end{tabular}

curvature (localized on the greater curvature and encircling) and Borrmann type 4 cancers. These results were quite similar to the findings of a previous report [7].

For complete removal of possible lymph node metastases at the splenic hilus, total gastrectomy with D2 lymph node dissection accompanied by pancreaticosplenectomy had been recommended as the classic surgical procedure for advanced proximal gastric cancer in Japan. However, previous reports including ours demonstrated that combined resection of distal pancreas did not improve the survival but actually resulted in a higher incidence of postoperative severe complications, such as pancreatic fistula, subphrenic abscess and postoperative diabetes [2022]. Maruyama et al. introduced pancreas-preserving splenectomy [20], and pancreas-preserving splenectomy has been substituted for pancreaticosplenectomy.

Many recent studies, however, demonstrated that simultaneous splenectomy did not have an effect on the survival rate of gastric cancer patients undergoing total gastrectomy, and it sometimes caused severe complications [10-12, 15]. Instead, Griffith et al. [17] advocate preservation of the spleen during surgery for gastric cancer because their retrospective study demonstrated that 
splenectomy was an independent predictor of poor survival after surgery. Recently, Yu et al. [16] reported a randomized controlled trial showing that prophylactic splenectomy had no impact on survival in patients with metastatic lymph nodes at the splenic hilus or in those with metastatic lymph nodes along the splenic artery. According to these findings, it would be better to abandon routine splenectomy for patients with advanced proximal gastric cancer. We, however, found some gastric cancer patients with pathologically proven metastasis at the splenic hilus who were completely cured by total gastrectomy with simultaneous splenectomy in this series, suggesting that splenectomy should be considered only for those patients who would obtain considerable benefits from it.

These findings prompted us to examine the gastric cancer patient subgroups that received survival benefit from lymph node dissection at the splenic hilus by splenectomy. It has been recognized that a reasonable analytical approach is to estimate the survival benefit of lymph node dissections using an index calculated by multiplying the incidence of lymph node metastasis by the 5-year survival rate of patients with metastasis at that station [5]. Therefore, we calculated the index with respect to splenic hilar metastasis in subgroups of patients with each clinicopathological factor. Accordingly, the overall index was 5.49, and the index was more than 10 in patient subgroups with primary tumors localized on the greater curvature (19.4) and Borrmann type 4 cancers (12.9). The higher index in primary tumors localized on the greater curvature resulted from both a higher incidence and a relatively favorable prognosis. Although the number in the patient subgroups was small, all patients with splenic hilar metastasis survived more than 5 years. With respect to Borrmann type 4 cancers, the 5-year survival was exceptionally high in the present study, possibly owing to strict selection criteria such as the avoidance of cases with metastasis to the para-aortic nodes and those with direct invasion of the spleen and the pancreas. Survival of patients with type 4 gastric cancer has generally been considered to be worse than that of the present study; however, the high index would be derived from the high incidence of metastasis. That splenectomy is beneficial to the patients with type 4 cancer might apply only to the institutions where the 5-year survival rate for type 4 cancer is relatively favorable.

Because this retrospective study did not include patients who underwent spleen-preserving gastrectomy, it remains uncertain if splenectomy has a prognostic impact on patients with advanced proximal gastric cancer. Moreover, the negative aspect of splenectomy has not yet been examined immunologically in detail. Consequently, it is difficult to judge whether the overall index (5.49) calculated in the present study represents prognostic significance or not. The precise impact of prophylactic splenectomy on the prognosis should be determined by future randomized controlled trials. The Japan Clinical Oncology Group (JCOG) 0110 randomized controlled trial is currently in progress [23].

\section{Conclusion}

Although the precise impact of splenectomy on prognosis remains uncertain, the present study suggests that patients with primary tumors localized on the greater curvature and Borrmann type 4 cancers might obtain a relatively high survival benefit from lymph node dissection at the splenic hilus by splenectomy.

\section{References}

1. Bonenkamp JJ, Hermans J, Sasako M, van de Velde CJH. Extended lymph-node dissection for gastric cancer. N Engl J Med. 1999;340:908-14.

2. Cuschieri A, Weeden S, Fielding J, Bancewicz J, Craven J, Joypaul V, et al. Patient survival after D1 and D2 resections for gastric cancer: long-term results of the MRC randomized surgical trial. Br J Cancer. 1999;79:1522-30.

3. Sano T, Sasako M, Yamamoto S, Nashimoto A, Kurita A, Hiratsuka M. Gastric cancer surgery: morbidity and mortality results from a prospective randomized controlled trial comparing D2 and extended para-aortic lymphadenectomy-Japan Clinical Oncology Group study 9501. J Clin Oncol. 2004;22:2767-73.

4. Sasako M, Sano T, Yamamoto S, Kurokawa Y, Nashimoto A, Kurita A, et al. D2 lymphadenectomy alone or with para-aortic nodal dissection for gastric cancer. N Engl J Med. 2008;359: 453-62.

5. Sasako M, McCulloch P, Kinoshita T, Maruyama K. New method to evaluate the therapeutic value of lymph node dissection for gastric cancer. Br J Surg. 1995;82:346-51.

6. Ishikawa S, Shimada S, Miyanari N, Hirota M, Takamori H, Baba $\mathrm{H}$. Pattern of lymph node involvement in proximal gastric cancer. World J Surg. 2009;33:1687-92.

7. Mönig SP, Collet PH, Baldus SE, Schmackpfeffer K, Schröder $\mathrm{W}$, Thiele $\mathrm{J}$, et al. Splenectomy in proximal gastric cancer: frequency of lymph node metastasis to the splenic hilus. J Surg Oncol. 2001;76:89-92.

8. Ikeguchi M, Kaibara N. Lymph node metastasis at the splenic hilum in proximal gastric cancer. Am Surg. 2004;70:645-8.

9. Shin SH, Jung H, Choi SH, An JY, Choi MG, Noh JH, et al. Clinical significance of splenic hilar lymph node metastasis in proximal gastric cancer. Ann Surg Oncol. 2009;16:1304-9.

10. Kunisaki C, Makino H, Suwa H, Sato T, Takashi O, Nagano Y, et al. Impact of splenectomy in patients with gastric adenocarcinoma of the cardia. J Gastrointest Surg. 2007;11:1039-44.

11. Sasada S, Ninomiya M, Nishizaki M, Harano M, Ojima Y, Matsukawa $\mathrm{H}$, et al. Frequency of lymph node metastasis to the splenic hilus and effect of splenectomy in proximal gastric cancer. Anticancer Res. 2009;29:3347-51.

12. Maehara Y, Moriguchi S, Yoshida M, Takahashi I, Korenaga D, Sugimachi K. Splenectomy does not correlate with length of survival in patients undergoing curative total gastrectomy for 
gastric carcinoma. Univariate and multivariate analyses. Cancer. 1991;67:3006-9.

13. Csendes A, Burdiles P, Rojas J, Braghetto I, Diaz JC, Maluenda F. A prospective randomized study comparing D2 total gastrectomy versus D2 total gastrectomy plus splenectomy in 187 patients with gastric carcinoma. Surgery. 2002;131:401-7.

14. Japanese Gastric Cancer Association. Japanese classification of gastric carcinoma, 2nd English edn. Gastric Cancer. 1998;1:10-24.

15. Otsuji E, Yamaguchi T, Sawai K, Ohara M, Takahashi T. End results of simultaneous splenectomy in patients undergoing total gastrectomy for gastric carcinoma. Surgery. 1996;120:40-4.

16. Yu W, Choi GS, Chung HY. Randomized clinical trial of splenectomy versus splenic preservation in patients with proximal gastric cancer. Br J Surg. 2006;93:559-63.

17. Griffith JP, Sue-Ling HM, Martin I, Dixon MF, McMahon MJ, Axon AT, et al. Preservation of the spleen improves survival after radical surgery for gastric cancer. Gut. 1995;36:684-90.

18. Okinaga $\mathrm{K}$, Iinuma $\mathrm{H}$, Kitamura $\mathrm{Y}$, Yokohata $\mathrm{T}$, Inaba $\mathrm{T}$, Fukushima R. Effect of immunotherapy and spleen preservation on immunological function in patients with gastric cancer. J Exp Clin Cancer Res. 2006;25:339-49.
19. Takahashi T, Sawai K, Hagiwara A, Takahashi S, Seiki K, Tokuda H. Type-oriented therapy for gastric cancer effective for lymph node metastasis: management of lymph node metastasis using activated carbon particles adsorbing an anticancer agent. Semin Surg Oncol. 1991;7:378-83.

20. Maruyama K, Sasako M, Kinoshita T, Sano T, Katai H, Okajima K. Pancreas-preserving total gastrectomy for proximal gastric cancer. World J Surg. 1995;19:532-6.

21. Otsuji E, Yamaguchi T, Sawai K, Okamoto K, Takahashi T. End results of simultaneous pancreatectomy, splenectomy and total gastrectomy for patients with gastric carcinoma. Br J Cancer. 1997;75:1219-23.

22. Otsuji E, Yamaguchi T, Sawai K, Okamoto K, Takahashi T. Total gastrectomy with simultaneous pancreaticosplenectomy or splenectomy in patients with advanced gastric carcinoma. Br J Cancer. 1999;79:1789-93.

23. Sano T, Yamamoto S, Sasako M. Randomized controlled trial to evaluate splenectomy in total gastrectomy for proximal gastric carcinoma: Japan Clinical Oncology Group study JCOG 0110-MF. Jpn J Clin Oncol. 2002;32:363-4. 